\title{
Scenarios for Fleet Assignment: A Case Study at Lion Air
}

\author{
Fried Markus Allung Blegur ${ }^{1}$, Toni Bakhtiar ${ }^{2}$, Amril Aman $^{3}$ \\ ${ }_{1,2,3}$ (Departemen of Mathematics, Bogor Agricultural University, Indonesia)
}

\begin{abstract}
Given the sets of flights and aircrafts of an airline, the fleet assignment problem consists of assigning the most profitable aircraft in every flight. In this paper, the model of fleet assignment is set up using the data from the airline company which has the largest market in Indonesia, i.e. Lion Air. It's involved the runway constraints in the model to result more realistic scenarios, where three scenarios of the fleet assignment have been analyzed. The aim of the first scenario is to assign the most appropriate fleet type to flights while minimizing the cost. The second scenario is to see what is the minimum number of aircraft required to cover all flights. The aim of the third scenario is to assign the most appropriate fleet type to flights while minimizing not only the cost but also the number of aircraft for all flights. Models have been set up under constraints of all airline operations and formulated in term of an integer linear programming. The solution of these problems generates a minimum daily cost of fleet assignment and the minimum number of aircraft for all flights.
\end{abstract}

Keywords: Cost minimization, fleet assignment, integer linear programming, lion air, number of aircraft

\section{Introduction}

The problem of fleet assignment is one of the hardest and most comprehensive problems faced in airline planning, where airlines typically operate a number of different fleet types. Each fleet type has different characteristics and costs, such as seating capacity, landing weights, crew, maintenance, and fuel [9]. Consequently the assignment of each fleet type will give different costs. Assigning fleet types to flight legs effectively is crucial in airline planning because the objective is to minimize cost to the airline. The goal of fleet assignment is to assign as many flight segments as possible in a schedule to one or more fleet types, while optimizing some objective function and meeting various operational constraints [1]. This planning concerns only fleet type, not a particular aircraft.

In fleet assignment, profit is maximized by minimizing two types of costs: operational and spill costs [8]. Operational costs are those for flying the flight leg with the assigned aircraft type and usually include such things as fuel cost, landing fees, depreciation and amortization and passenger service cost [5]. Spill costs represent lost opportunity costs that arise if passenger demand exceeds the aircraft capacity and, thus, potential revenue is lost [3]. An optimum solution is found in [4] using the basic Fleet Assignment Model (FAM). FAM had been used in the case study in Turkey, where the study uses real data of Turkish Airlines [7].

In this paper, the FAM will be modified and applied to the largest private airline in Indonesia, Lion Air. There are three scenarios to be explored. The first scenario presents the best fleet assignment for each flight leg that gives the minimum cost of the airline. In the second scenario, the objective function is modified to minimize the total number of aircraft to cover all flights in schedule. The third scenario presents the best fleet assignment for each flight leg that gives the minimum cost of the airline while minimize the total number of aircraft to cover all flights in schedule.

\section{Fleet Assignment Model}

The aircraft assignment is the process that defines which aircraft will perform each scheduled flight. The first step of this process was the fleet assignment which aims to find the profit maximizing assignment of aircraft types to flight legs in the schedule without exceeding the available aircrafts and ensuring balance of aircraft type at each airport location each day. Wherever possible, the goal is to match as closely as possible seat capacity with passenger demand for each flight leg.

The following model, referred to as the fleet assignment model with runway constraints, is a modified version of FAM proposed by [6]. We define the following sets, parameters, and variables. We denote by $F$ the set of flights $(i \in F)$, by $K$ the set of fleet types $(j \in K)$, by $C$ the set of last-nodes, representing all nodes with aircraft grounded overnight at an airport in the network $(k \in C)$, by $c_{i, j}$ the cost of assigning fleet type $j$ to flight $i$, by $N_{j}$ the number of available aircraft in fleet type $j$, by $M$ the number of nodes in the network, and by

$s_{i, j}=\left\{\begin{array}{r}1 \text { if flight } i \text { is an arrival at node } k \\ -1 \text { if flight } i \text { is a departure from node } k\end{array}\right.$

Decision Variables:

$x_{i, j}=\left\{\begin{array}{l}1 \text { if flight } i \text { is assigned to fleet }- \text { type }\} \\ 0 \text { otherwise }\end{array}\right.$ 
$G_{k, j}$ is integer decision variable representing number of aircraft of fleet-type $j$ on ground at node $k$.

The objective function of this problem is to minimize the total daily cost of assigning the various available fleet types to all the flights in the schedule, i.e.

$\min \sum_{j \in R} \sum_{i \in F} c_{i, j} x_{i, j}$

Under constraints:

1. The flight cover constraint to ensure that each flight is flown by one type of fleet.

$\sum_{j \in K} x_{i, j}=1 ; \forall i \in F$.

2. The aircraft balance constraint to ensure that an aircraft of the right fleet type will be available at the right place at the right time.

$G_{k-1, j}+\sum_{i \in F} s_{i, j} x_{i, j}=G_{k, j} \forall \forall \in M, \forall j \in K$.

3. The fleet size constraint to ensure that the number of aircraft within each fleet does not exceed the available fleet size.

$\sum_{k \in C} G_{k, j} \leq N_{j j} ; \forall j \in K$.

4. Decision variable $x_{i, j}$ is a binary

$x_{i, j} \in\{0,1\} ; \forall \in F, \forall j \in K$.

5. Decision variable $G_{k, j}$ is an integer

$G_{k, j} \in Z^{+} ; \forall k \in M, \forall j \in K$.

According to Federal Aviation Administration (FAA) guidance, Advisory Circular 150/5325-4B, Runway Length Requirements for Airport Design, an aircraft can take off at airport that have runways longer than the minimum length requirements [2]. We denote by $o_{i}$ the origin airport runway length to flight $i, d_{i}$ the destination airport runway length to flight $i$, and $r_{j}$ the minimum airport runway length requirements to take off and landing fleet type $j$.

6. Take off runway constraint: an aircraft can only be assigned to airports that have runways longer than the minimum length of runway required the aircraft to take off.

$\sum_{j \in T} r_{j} x_{i, j} \leq o_{i} ; \forall i \in F$.

7. Since the aircraft will take off from the destination airport to the next flight, landing runway constraint is $\sum_{j \in T} r_{j} x_{i, j} \leq d_{i} ; \forall i \in F$

\section{Scenarios}

We consider three scenarios which represented by following objective functions:

1. The aim of first scenario is to assign the most appropriate fleet type to flights while minimizing the assignment cost. The objective function is

$\min \sum_{j \in \mathbb{K}} \sum_{i \in F} c_{i, j} x_{i_{j}}$

2. The second scenario seeks the minimum number of aircraft to cover all flights, i.e.,

$\min \sum_{k \in C} G_{k j}$

3. The aim of the third scenario is to assign the most appropriate fleet type to flights while minimizing not only the assignment cost but also the number of aircraft for all flights. This scenario is easily solved by using the results of the second scenario. Suppose we denote by $N^{*}$ the minimum number of aircraft to cover all flights, then this scenario can be performed by

$\min \sum_{j \in R} \sum_{i \in F} c_{i, j} x_{i, j}$

under an additional constraint 


$$
\sum_{j \in T} \sum_{k \in C} G_{k, j} \leq N^{*} .
$$

\section{Application To Lion Air}

Lion Air serves 605 domestic flights to 34 origin-destination cities and 26 international flights to/from four cities in foreign countries using 96 aircraft from 5 fleet types available. Airport codes and airport runway length are presented in Table 1. Some of the flight schedule route (all of 631 flights per day), is presented in Table 2. Fleet types, the number of aircraft, seat capacity, operating cost, and the minimum runway length of the airport required to take off every fleet-type are presented in Table 3. Demand in Table 2 was calculated from the flight frequency, seat capacity, load factor, market share and passenger growth.

Table 1. Airport code and airport runway length served by Lion Air

\begin{tabular}{cccccc}
\hline Airport Code & Runway $(\mathrm{m})$ & Airport Code & Runway $(\mathrm{m})$ & Airport Code & Runway $(\mathrm{m})$ \\
\hline AMQ & 2,501 & KDI & 2,250 & PNK & 2,250 \\
BPN & 2,495 & KOE & 2,501 & SRG & 2,680 \\
BTJ & 2,501 & LOP & 2,750 & SOC & 2,600 \\
TKG & 2,501 & UPG & 3,100 & SUB & 3,001 \\
BDO & 2,250 & MDC & 2,651 & TNJ & 2,250 \\
BDJ & 2,501 & KNO & 3,003 & TRK & 2,250 \\
BTH & 4,040 & MKQ & 2,501 & TTE & 2,100 \\
BKS & 2,239 & PDG & 2,750 & JOG & 2,200 \\
DPS & 3,001 & PKY & 2,501 & JED & 3,299 \\
GTO & 2,501 & PLM & 3,001 & KUL & 4,124 \\
CGK & 3,661 & PLW & 2,251 & PEN & 3,352 \\
DJB & 2,220 & PGK & 2,250 & SIN & 2,748 \\
DJJ & 2,501 & PKU & 2,240 & & \\
\hline
\end{tabular}

In this network, the hubs selected are CGK, SUB, UPG and BTH, while other airports are the spokes.

Table 2. Flight schedule, the distance between the airports, and demand

\begin{tabular}{cccccccc}
\hline No. & Flight no. & Origin & Destination & Departure & Arrival & Distance (mile) & Demand \\
\hline 1 & 694 & CGK & SUB & $0: 30$ & $2: 00$ & 424 & 216 \\
2 & 798 & UPG & DJJ & $0: 40$ & $4: 05$ & 1457 & 210 \\
3 & 790 & CGK & AMQ & $1: 30$ & $5: 00$ & 1487 & 218 \\
$\cdot$ &. &. &. &. &. &. &. \\
. &. &. &. &. &. &. &. \\
. &. &. &. &. &. &. &. \\
631 & 826 & UPG & TTE & $2: 30$ & $4: 20$ & 677 & 153 \\
\hline
\end{tabular}

Table 3. Fleet characteristics

\begin{tabular}{ccccc}
\hline Fleet Type & Number of Aircraft & Seat Capacity & Cost $(\$)^{\mathrm{a}}$ & Runway $(\mathrm{m})$ \\
\hline B733 & 3 & 149 & 3,283 & 1,600 \\
B734 & 3 & 168 & 3,283 & 2,000 \\
B738 & 21 & 189 & $3,536.54$ & 2,300 \\
B739 & 67 & 213 & $3,233.05$ & 2,300 \\
B744 & 2 & 505 & $9,443.76$ & 3,300 \\
\hline
\end{tabular}

${ }^{\mathrm{a} O p e r a t i n g ~ c o s t ~ p e r ~ h o u r ~}$

The assignment cost $c_{i, j}$ consists of operating and spill costs, where

1. Operating Cost $=$ Operating Cost per Hour $\times$ Flight Duration,

2. Spill Cost $=$ Passenger-Spill $\times$ RASM $\times$ Distance.

RASM is Revenue per Available Seat Mile or 'unit revenue' which represents how much an airline made across all the available seats that were supplied.

\subsection{Scenario to Scenario 1}

The linear programming for this scenario has 9,465 variable (3,155 binary and 6,310 integer) and 7,653 constraints. Using optimization software, the solution to this scenario generates a minimum daily cost of fleet assignment of $\$ 3,602,545.60$. Table 4 shows the number of aircrafts for each fleet type staying overnight at certain airports. 
Table 4. The number of aircraft grounded overnight at each airport for Scenario 1

\begin{tabular}{|c|c|c|c|c|c|}
\hline \multirow{2}{*}{$\begin{array}{c}\text { Number of } \\
\text { Aircraft }\end{array}$} & \multicolumn{5}{|c|}{ Fleet Type } \\
\hline & B733 & B734 & B738 & B739 & B744 \\
\hline 1 & $\begin{array}{l}\text { TKG, } \\
\text { UPG, } \\
\text { KNO }\end{array}$ & CGK & $\begin{array}{l}\text { BPN, BTJ, BDO, BTH, LOP, } \\
\text { MDC, KNO, PLW, PNK, SOC, } \\
\text { TRK, TTE, JOG, KUL }\end{array}$ & $\begin{array}{l}\text { TKG, BKS, GTO, DJB, DJJ, } \\
\text { KOE, LOP, KNO, PDG, PLM, } \\
\text { PLW, PKU, SRG, JOG, KUL }\end{array}$ & $\begin{array}{l}\text { CGK, } \\
\text { KNO }\end{array}$ \\
\hline 2 & & BTH & SUB & DPS, PKY, TRK & \\
\hline 3 & & & & BDJ, MDC & \\
\hline 4 & & & & BPN, SUB & \\
\hline 5 & & & CGK & UPG & \\
\hline 27 & & & & CGK & \\
\hline
\end{tabular}

\subsection{Solution to Scenario 2}

The linear programming for this scenario involves the same number of variables and constraints. The assignment cost in this scenario is $\$ 3,900,573.05$, it is $\$ 298,027.45$ more expensive than that of the first scenario. Table 5 shows the number of aircraft for each fleet type staying overnight at certain airports. It is provided that 95 units of aircraft are required to complete the task with one unit of B739 is idle.

Table 5. The number of aircraft grounded overnight at each airport for Scenario 2

\begin{tabular}{cllll}
\hline $\begin{array}{c}\text { Number of } \\
\text { Aircraft }\end{array}$ & \multicolumn{3}{c}{ Fleet Type } & \multicolumn{1}{c}{ B739 } \\
\cline { 2 - 5 } 1 & B733 & B734 & \multicolumn{1}{c}{ B738 } & \multicolumn{1}{c}{ B744 } \\
\hline & BPN, & CGK, & TKG, BTH, BKS, GTO, & BTJ, TKG, BTO, BDJ, DJB, DJJ, KOE, KNO, \\
& CGK, & UPG, & MDC, PDG, SUB & PLM, PKU, PNK, SRG, SOC, TTE, KUL \\
2 & MDC & SUB & & KUL \\
3 & & & BPN, BDJ, UPG, KNO & BPN, BTH, DPS, LOP, MDC, PKY, PLW, JOG \\
4 & & & & UPG, TRK \\
6 & & & CGK & CGK \\
25 & & & &
\end{tabular}

\subsection{Solution to Scenario 3}

In this scenario, the number of constraints increases to 7,654. By making an upper bound to the minimum number of required aircraft of $N^{*}=95$ and organizing the fleet assignment, we can attain the daily cost of $\$ 3,612,300.14$, which is $\$ 288,272.91$ cheaper than that of Scenario 2 . The number of aircraft for each fleet type staying overnight at each airport are as shown in Table 6.

Table 6. The number of aircraft grounded overnight at each airport for Scenario 3

\begin{tabular}{cccll}
\hline \multirow{2}{*}{$\begin{array}{c}\text { Number of } \\
\text { Aircraft }\end{array}$} & \multicolumn{3}{c}{ Fleet Type } \\
\cline { 2 - 5 } & B733 & B734 & \multicolumn{1}{c}{ B738 } & \multicolumn{1}{c}{ B739 } \\
\hline & TKG, & CGK & BPN, BTJ, BDO, BTH, LOP, UPG, & TKG, BKS, GTO, DJB, DJJ, \\
& UPG, & & MDC, KNO, PLW, PNK, SOC, SUB, & KOE, LOP, PDG, PLM, PLW, \\
2 & KNO & & TRK, TTE, JOG, KUL & PKU, SRG, JOG, KUL \\
3 & & BTH & & DPS, KNO, PKY, TRK \\
4 & & & & BDJ, MDC \\
5 & & & CGK & BPN, UPG \\
26 & & & & SUB \\
\hline
\end{tabular}

\section{Conclusion}

In this paper, the fleet assignment problem in real cases for the largest private airline in Indonesia, Lion Air, was studied. In addition to the basic model, we consider the length of airport runway as constraints. The aims were minimizing the cost and number of aircrafts. We have the following conclusions:

1. The best results of fleet assignment to each flight leg in the schedule gives the minimum cost of $\$ 3,602,545.60$. This means that the different assignments will provide a greater cost impact. Assignment involves all the aircrafts available (96 aircrafts).

2. The minimum number of aircrafts to cover all flights is 95 with a B739 is not used. This aircraft will be located in one of the hubs during the night for parking, nightly maintenance, and preparation to other destinations. Since the model aims to solely minimize the number of required aircrafts, it is obvious that the operation of a big and thus costly aircraft, i.e. a B744, is very high. Indeed, this increases the daily cost up to $8.27 \%$.

3. The model can be modified to obtain the minimum assignment cost while minimizing the number of aircraft required covering all flights in the schedule. By limiting the number of aircrafts up to 95 units, we can rearranging the fleet assignment such that reducing the daily cost. This option can be considered when it is required to ground an aircraft for maintenance. 


\section{References}

[1] J. Abara, Applying integer linear programming to the fleet assignment problem, Interfaces, 19(4), 1989, $20-28$.

[2] [ACRP] Airport Cooperative Research Program, Federal Aviation Administration, Improved models for risk assessment of runway safety areas (Washington DC, Transportation Research Board, 2011).

[3] C. Barnhart, P.P. Belobaba, and A.R. Odoni, Applications of operations research in the air transport industry, Transportation Science, 37 (4), 2003, 368-391.

[4] M. Bazargan, Airline operations and scheduling. 2nd ed (United States, Ashgate, 2004).

[5] P. Belobaba, A. Odoni, and C. Barnhart, The global airline industry (United States, Wiley, 2009).

[6] C.A. Hane, C. Barnhart, E.L. Johnson, R.E. Marsten, G.L. Nemhauser, and G. Sigismondi, The fleet assignment problem: solving a large-scale integer program, Mathematical Programming, 70, 1995, 211-232.

[7] Y. Ozdemir, H. Basligil, and B. Sarsenov, A large scale integer linear programming to the daily fleet assignment problem: a case study in Turkey, Procedia-Social and Behavioral Sciences, 62, 2012, 849-853.

[8] R. Subramanian, R.P. Scheff Jr., J.D. Quillinan, D.S. Wiper, and R.E. Marsten, Coldstart: fleet assignments at Delta Air Lines, Interfaces, 24(1), 1994, 104-120.

[9] G. Yu, and B. Thengvall, Airline optimization, in P.M. Pardalos and M.G.C. Resende (Ed.), Handbook of Applied Optimization, 2(18) (New York: Oxford University Pr., 1999) 689-703. 